\title{
Power-free bistable threshold accelerometer made from a carbon nanotube framework
}

\author{
J. D. Tanner and B. D. Jensen \\ Brigham Young University, Department of Mechanical Engineering, Provo, Utah, USA \\ Correspondence to: B. D. Jensen (bdjensen@byu.edu)
}

Received: 20 March 2013 - Revised: 8 July 2013 - Accepted: 27 July 2013 - Published: 16 December 2013

\begin{abstract}
This paper presents a design and a method for fabricating and testing fully compliant, bistable threshold accelerometers made from a carbon-infiltrated carbon nanotube framework. Two different configurations based on differences in compliant beam angles are presented. Both configurations were tested under constant accelerations produced by mounting them on a spinning disk and under impulse accelerations by mounting them to the end of a swinging pendulum with a stop at the lowest point of the pendulum path. Tests were also performed to observe the potential effects of stress relaxation in the carbon nanotube material. This was done by placing the accelerometers in their second stable position (nonfabricated position) and then testing them after a period of $24 \mathrm{~h}$. Results show that in eight of the twelve tests there was no significant change due to stress relaxation. In the other four tests, the change was relatively small, especially when compared to stress relaxation effects in other materials such as plastics. Measurements indicate that the accelerometers show very high repeatability individually. However, we also observed that there is significant variation in switching acceleration between accelerometers with identical geometric parameters. This may be due to random variation occurring during the fabrication process.
\end{abstract}

\section{Introduction}

Recent developments in the field of microelectromechanical systems (MEMS) based accelerometers have proven highly useful in many applications. Laptop computers are often monitored by accelerometers to protect hard drives in the event of a drop or severe jolt (Yuan et al., 2010). Airbag systems in most automobiles are deployed when a capacitive accelerometer detects an impact above a given threshold (Zhao et al., 2007). Many shipping companies are interested in tracking the delivery of packages to detect any severe impacts that may occur during transit (Sun et al., 1997). And recently, earthquake early warning systems have been implemented using threshold accelerometer networks described by Zollo et al. (2010).

However, most of these applications utilize accelerometers that require power to operate as the circuits that they are integrated into continually gather and process data. By creating a bistable accelerometer that responds to a particular acceleration threshold, no power is required. One design, developed by Selvakumar et al. (2001), incorporates a lowpower circuit to interface with their threshold accelerometers and communicates only in the event of an acceleration beyond the designed threshold, thus conserving power. Experiments performed by Todd et al. (2008) show that similar types of accelerometers can be used in conjunction with passive RFID technology to relay information to an external reader. For example, accelerometers could easily be used to detect threshold levels of impact experienced by athletes in many sports. One study which measured the accelerations experienced by collegiate football players revealed that $40 \%$ of impacts that were measured were greater than $20 \mathrm{~g}$ 's (Rowson et al., 2009). This type of technology would allow coaches or staff to take preventative action when impacts are detected that may be unsafe.

The intent of our work was to successfully fabricate and test a bistable accelerometer made of carbon-infiltrated carbon nanotube (CI-CNT) material. The accelerometer is capable of accurately responding to threshold levels of acceleration in a single direction in a repeatable manner. Other 
threshold accelerometer designs have been demonstrated by Ma et al. (2003) and Churaman et al. (2008), which use a proof mass between flexural members and is modeled as a mass-spring system. However, these models are not bistable; rather, they create an electrical contact upon reaching the threshold acceleration. A cantilever design has also been presented by Loke et al. (1991), Genberg and Pecht (1989), and Edmans et al. (1997), which fixes a proof mass on one end with sensing elements beneath the cantilever beam to detect deflection indicating a certain acceleration. All of these examples use micromachining techniques for accelerometer fabrication but do not use CI-CNT material like those described in this paper.

The use of CI-CNT material proves highly advantageous in many MEMS applications because of its exceptionally high strain fracture characteristics. Many MEMS rely on compliant motion for their functionality. Fazio et al. (2011) measured the maximum strain of CI-CNT structures and compared it to that of other typical MEMS materials. One common material used in MEMS fabrication is polysilicon, which is capable of enduring approximately $0.7 \%$ strain before fracture. In comparison, they found that the CI-CNT material was able to withstand greater than $2 \%$ strain prior to fracture.

In addition to favorable material properties for compliant motion, CI-CNT accelerometers can be produced much smaller than those that have been produced previously using plastics or metals, such as those described by Hansen et al. (2007). For example, the plastic mechanisms reported by Hansen et al. (2007) had a leg length of $6.1 \mathrm{~mm}$, while the metal mechanisms reported by Todd et al. (2010) had a minimum length of $20.5 \mathrm{~mm}$. By comparison, the leg length used in this paper is $1 \mathrm{~mm}$. Moreover, the designs presented here were fabricated using low-cost film masks with a minimum resolution of about $15 \mu \mathrm{m}$. Using a higherresolution mask would allow further size reduction of at least 5 times. In addition, because CI-CNT structures are fabricated through traditional microfabrication techniques, high resolution is achieved in compliant elements of the sensor, resulting in higher accuracy in sensing the expected accelerations. These structures also have very high aspect-ratios making them very robust as demonstrated by Fazio et al. (2011) and Hutchison et al. (2010).

Lastly, inherent to bistable mechanisms is the fact that they can be interpreted as a binary output. They are either in one position or the other, not somewhere in between. A threshold accelerometer is designed to retain its state after it has been switched. By design, the force required to move from the initial state to the switched state is much higher than the force needed to move from the second position back to the fabricated position. Therefore, in most applications the sensor would be set to the second position until a threshold level of acceleration is experienced. At this point the sensor would switch back to the fabricated position allowing a user or electronic interface to detect this change.

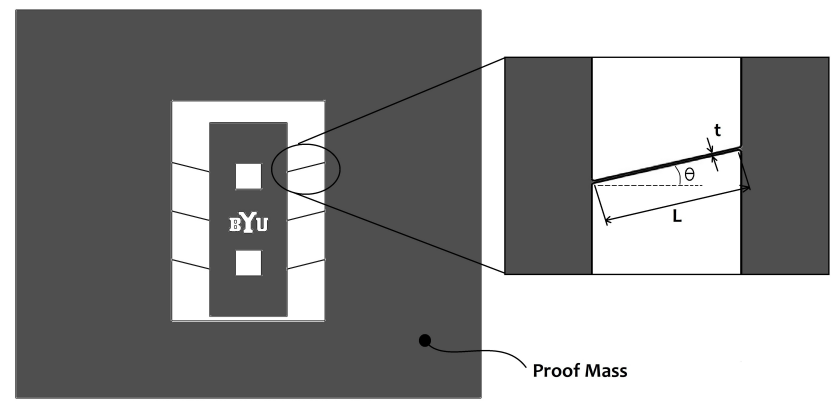

Figure 1. Bistable accelerometer design showing parameters that affect bistability and force required for switching.

This paper explores the design, fabrication, and testing of bistable threshold accelerometers fabricated using the highstrain method demonstrated by Fazio et al. (2011). The design is based on that presented by Todd et al. (2010). We show that the combination of the design and fabrication method results in threshold accelerometers with relatively small size, low stress relaxation, and high repeatability.

\section{Methods}

\subsection{Design}

Figure 1 shows a top view of the accelerometer design. As shown, the accelerometers consist of a proof mass and a middle section connected by the flexural beams. The center section is designed to be fixed by placing it on two electrical header pins. The proof mass is able to move vertically as the flexural beams buckle into the second stable position (or vice-versa). We designed two accelerometer configurations with six flexural beams, three on either side of the center section. In theory, only two beams are needed to produce bistability in the mechanism; however, by incorporating at least four beams into the accelerometer, the design provides greater torsional stiffness and is less prone to rotation about any axis. We chose to use six beams to provide for greater robustness.

To model and analyze the bistable accelerometers we used an elliptic integral solution derived by Todd et al. (2010). The solution provides an analytical calculation of forces and displacement of bistable mechanisms utilizing long, thin flexural beams. The force required to induce buckling in the flexure is dependent on flexure thickness, $t$, flexure length, $L$, flexure angle, $\theta$, material density, $\rho$ and modulus of elasticity, $E$. Using these parameters and the elliptic integral solution, we designed two different accelerometer configurations. Flexure angle, $\theta$, varied between the two configurations, one in which $\theta=8^{\circ}$ and the other where $\theta=14^{\circ}$. Flexure length, $L$, and flexure thickness, $t$, were designed to be the same in both configurations with values of $1 \mathrm{~mm}$ and $15 \mu \mathrm{m}$, respectively. The area of the proof mass also varied slightly between the designs with $A=97.5 \mathrm{~mm}^{2}$ and $A=97.7 \mathrm{~mm}^{2}$ for 


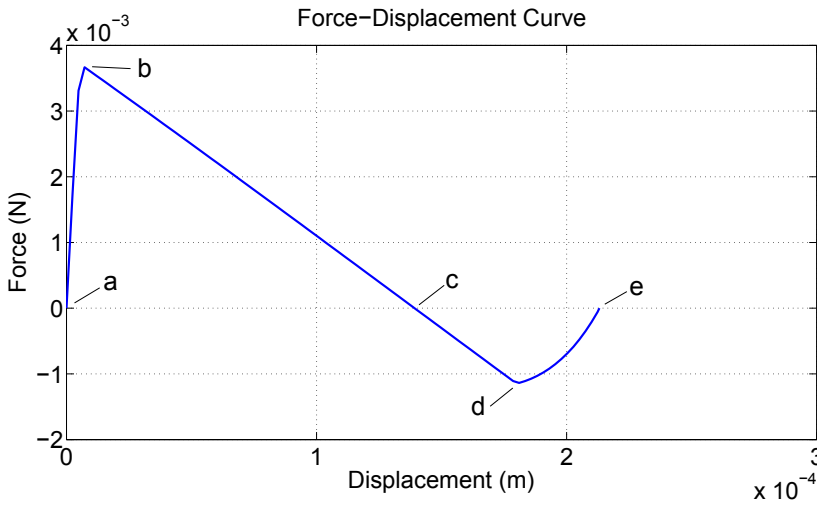

Figure 2. Force vs. displacement curve for a single flexural beam angled at $8^{\circ}$. (a) First stable position (fabricated position). (b) Switching force from position 1 to position 2. (c) Unstable equilibrium point. (d) Switching force from position 2 to position 1 . (e) Second stable position.

the 8 and $14^{\circ}$ designs, respectively. Because mass and required force both increase linearly with structure height (outof-page direction for Fig. 1), the switching acceleration does not change with a change in structure height according to the relation

$a=F / m$,

where $a$ is the calculated switching acceleration, $F$ is the total force, in Newtons, required for buckling in all six flexures, and $m$ is the proof mass. Therefore, this dimension was disregarded in the design. However, for sake of consistency, the accelerometers were grown to approximately $500 \mu \mathrm{m}$ when fabrication took place.

We assumed that the density would be constant in all accelerometers with a value of $\rho=2.4 \mathrm{~g} \mathrm{~cm}^{-3}$, based on preliminary testing. This value was used throughout the design of the accelerometers. However, measurements taken after fabrication suggest that there may be significant variability in the CNT material density due to random variation in the growth and infiltration process. The consequences of this variability are discussed further in Sect. 4.

Figures 2 and 3 show the analytical force-displacement curve for a single flexural beam from the 8 and $14^{\circ}$ configurations, respectively. The displacement information was used to appropriately design the space between the fixed portion and the proof mass to prevent contact during switching.

The accelerometers were designed to be manually switched into the second stable position. From the second stable position, an acceleration of sufficient magnitude applied along the switching direction would cause the mechanism to switch back to the first stable position. Because the switching force required to switch back again to the second stable state is much higher than that required to switch from position 1 to position 2 , the accelerometer would then tend to remain in its first stable state until its state is read. Hence, the

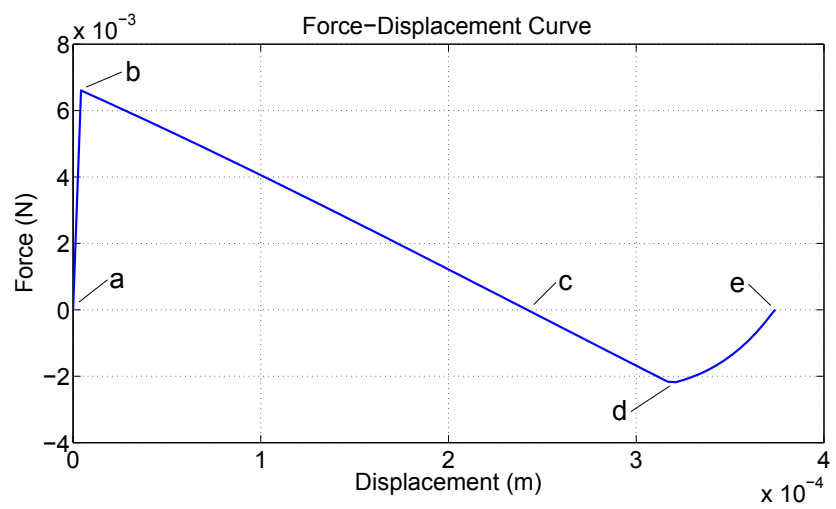

Figure 3. Force vs. displacement curve for a single flexural beam angled at $14^{\circ}$. (a) First stable position (fabricated position). (b) Switching force from position 1 to position 2. (c) Unstable equilibrium point. (d) Switching force from position 2 to position 1 . (e) Second stable position.

maximum force values for switching between the two stable points were also used in the design. Both configurations were designed to have approximately a $3: 1$ ratio of the absolute force required to switch from state 1 to state 2 and the absolute force to switch from state 2 back to state 1. Following reading, the accelerometer may be manually reset.

In other bistable mechanisms it may be advantageous to utilize a design in which switching forces required between stable states are equal. Lassooij et al. (2012) developed a statically balanced bistable gripper. Their design demonstrates this characteristic in which the unstable equilibrium point is positioned equidistant from either stable position.

\subsection{Fabrication}

The wafer preparation, nanotube growth, and infiltration processes that we used are similar to those described by Fazio et al. (2011) and Hutchison et al. (2010). An illustration of this process is given in Fig. 4.

Accelerometer fabrication begins by lithographically patterning the designs on a silicon wafer covered in alumina (Fig. $4 \mathrm{a}$ and $\mathrm{b}$ ). Iron is then deposited on the patterned wafer via thermal deposition to a thickness of $4 \mathrm{~nm}$ (Fig. 4c). The iron layer serves as the nanotube growth catalyst and the alumina layer prevents the iron from diffusing into the silicon during growth. After iron deposition the patterned photoresist is removed and the wafer is cut into smaller sections ready for nanotube growth and infiltration (Fig. 4d).

Growth and infiltration are performed in a one inch quartz tube in order to control the flow of gases. Nanotube growth accelerometers takes place in a furnace at $750{ }^{\circ} \mathrm{C}$. While the furnace is ramping up to temperature, hydrogen gas $\left(\mathrm{H}_{2}\right)$ flows at a rate of $232 \mathrm{sccm}$ in order to reduce the iron oxide that forms between photolithography and growth steps. Once the furnace reaches $750^{\circ} \mathrm{C}$, hydrogen and ethylene $\left(\mathrm{C}_{2} \mathrm{H}_{4}\right)$ 
(a)
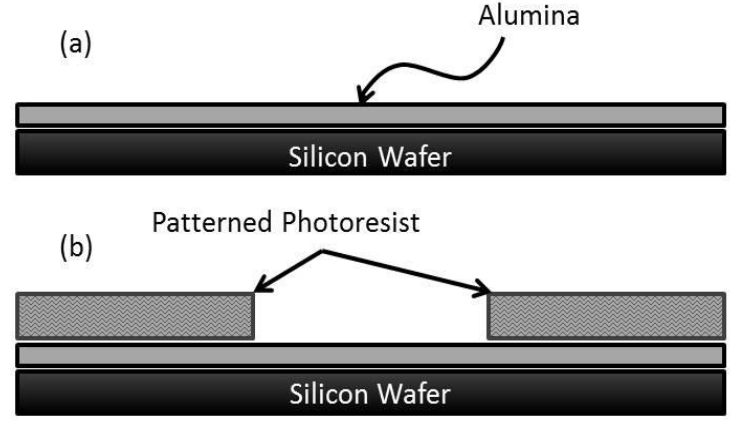

(c)

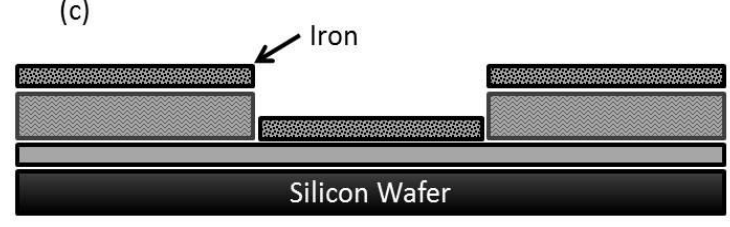

(d)
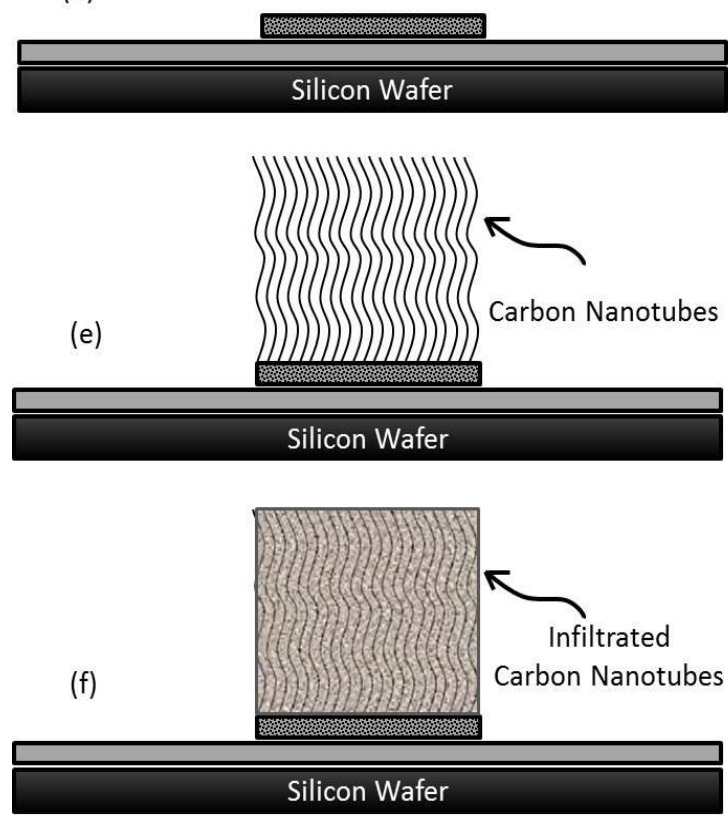

(g)

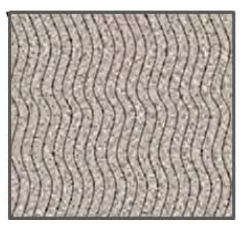

Figure 4. (a) Alumina is deposited ( $30 \mathrm{~nm})$ via PECVD to prevent iron diffusion into the silicon. (b) A positive photoresist is spun onto the wafer and lithographically patterned. (c) Iron is deposited by thermal evaporation to a thickness of $4 \mathrm{~nm}$. (d) The remaining photoresist is removed along with the iron on top of it. (e) Carbon nanotubes grow on the patterned iron via chemical vapor deposition. (f) The nanotube forest is filled with amorphous carbon. (g) The final structure is then removed from the substrate for testing. flow simultaneously at a rate of 232 and $246.8 \mathrm{sccm}$, respectively, resulting in carbon nanotube growth (Fig. 4e). After four minutes, the gas flow is stopped and the temperature setting is increased to $900{ }^{\circ} \mathrm{C}$ for infiltration. During the ramp up time, argon (Ar) flows at a rate of $249.5 \mathrm{sccm}$. Argon is used because it will not react negatively with the carbon nanotube structures while the temperature increases. At $900^{\circ} \mathrm{C}$, hydrogen and ethylene flow at the same rates used during growth for $25 \mathrm{~min}$. This infiltrates the carbon nanotubes with a film of vapor-deposited carbon (Fig. 4f). The furnace is then turned off and cooled to room temperature and the accelerometers are removed. At this point the accelerometers must be removed from the silicon substrate. This is done using oxygen plasma etching followed by a wetetch in $\mathrm{KOH}$ solution until the structures are freed from the silicon (Fig. 4g). They are then rinsed in water and dried. Fabricated samples are shown in both positions in Fig. 5.

\subsection{Testing}

In order to test the functionality and accuracy of the accelerometers, two types of experiments were designed. One test measured the response of the accelerometer to a constant acceleration and the other test measured the response when an impulse acceleration was applied. These tests allowed us to measure the required switching accelerations for each accelerometer. In addition to the threshold measurements, we were also interested in measuring any changes that may occur due to stress relaxation as a result of retaining the accelerometer in the second (non-fabricated) position for an extended time.

\subsubsection{Constant acceleration testing}

A centrifuge was used to apply a constant acceleration. The accelerometers were individually tested on a stage that was mounted on a rotating disk. The stage consists of two electrical header pins that interface with the square holes shown in Figs. 1 and 5. The accelerometers were enclosed by the plastic pieces seen in Figs. 6 and 7 in order to avoid any adverse affects of wind while the disk is spinning. The centripetal acceleration experienced by the accelerometer was calculated using the equation

$a=r\left(\frac{2 \pi \omega}{60}\right)^{2}$,

where $a$ is the acceleration in $\mathrm{m} \mathrm{s}^{-2}, r$ is the radius of rotation in meters, and $\omega$ is the speed of rotation in rpm. The radius of rotation is $r=0.1 \mathrm{~m}$.

A total of six accelerometers were tested - three of each configuration. Each accelerometer was tested five times. This was done by manually switching the accelerometer from the fabricated state to the second stable state using a linear micrometer stage, then mounting it on the testing stage. The 


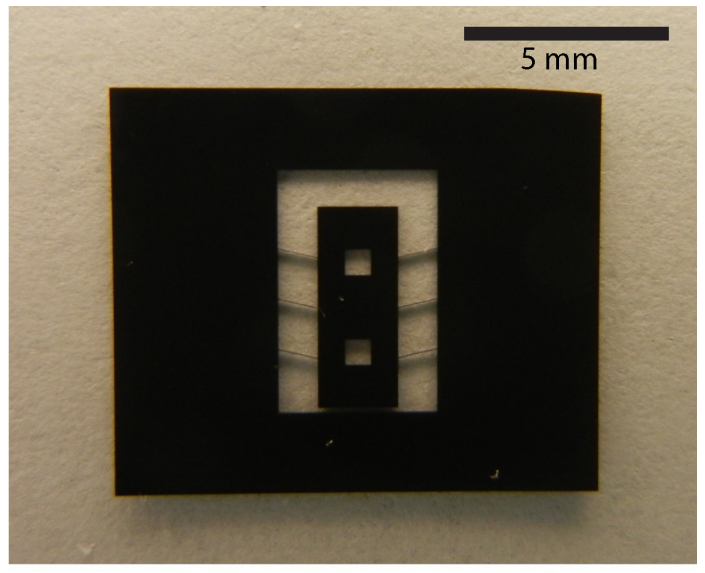

(a)

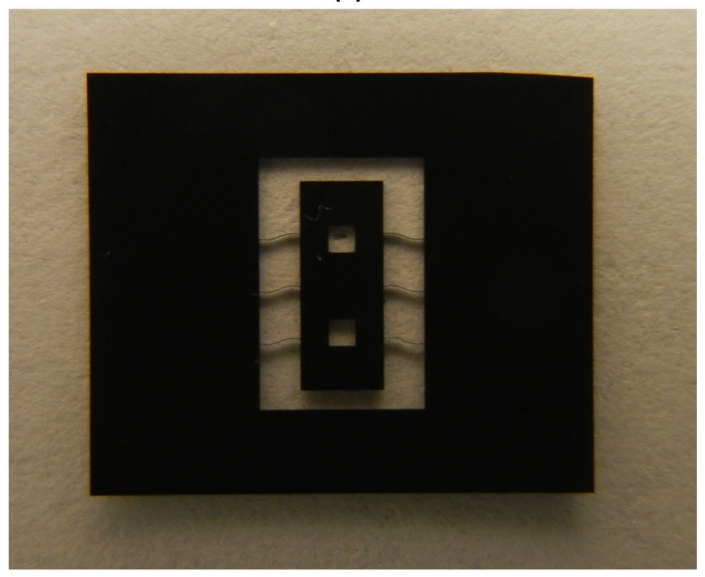

(b)

Figure 5. (a) $14^{\circ}$ accelerometer design in its fabricated position. (b) Accelerometer switched to its second stable position.

speed was increased incrementally in steps of approximately $2 \mathrm{rpm}$ until the accelerometer snapped back to state 1 . The switching acceleration, calculated from Eq. (1), was recorded and the process was repeated.

At the end of the fifth test, the accelerometer was again switched into state 2 and left in this position for a period of $24 \mathrm{~h}$. At the end of $24 \mathrm{~h}$ the accelerometer was tested five more times in the same way as the previous five tests. This was done to measure any effects of stress relaxation. In some cases, flexural beams would break during handling of the accelerometers between tests. In this case, the data for that accelerometer were saved but not included in the statistical analysis (except in one case in which the accelerometer broke on the last test; nine tests were deemed to still have statistical value). These partial data sets can be found in Appendix A.

\subsubsection{Impulse acceleration testing}

To test the accelerometers under impulse conditions, the same plastic stage with electrical header pins was used, but was mounted to a pendulum shown in Fig. 8. The impulse

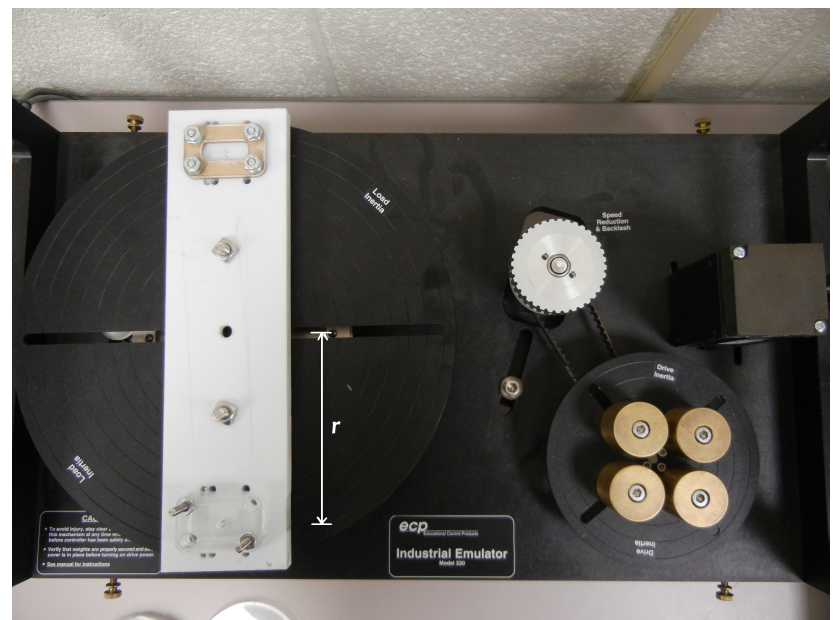

Figure 6. Test setup for applying constant centripetal accelerations.

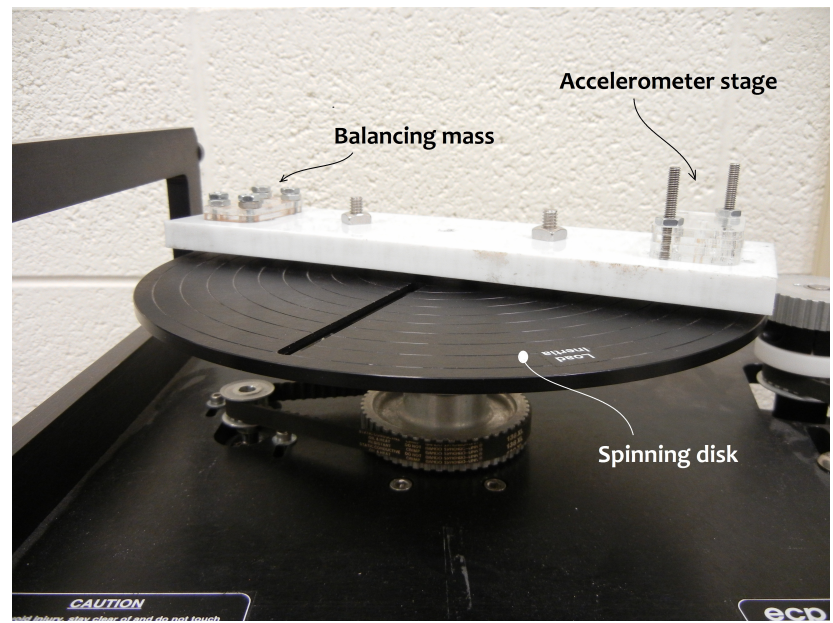

Figure 7. Disk and accelerometer stage used to test switching accelerations.

was generated by placing a stop at the lowest point of the pendulum path. A small, single-axis accelerometer (PCB Piezotronics, Model \#352B10) was mounted to the stage in order to measure the accelerations produced in the direction of motion of the proof mass. The pendulum was released from a starting position and the drop angle was increased in small increments until switching occurred. LabVIEW was used to record and store data during this time. The accelerations where switching took place could then be determined from the data. In order to accurately track the duration, amplitude, and overall shape of the impulse curve, sampling occurred at $10 \mathrm{kHz}$.

This type of testing is representative of many situations in which an accelerometer may be used. For example, dropping a package, a collision of two football helmets, and an automobile accident all produce impulse accelerations. However, depending on the materials involved and their elastic 


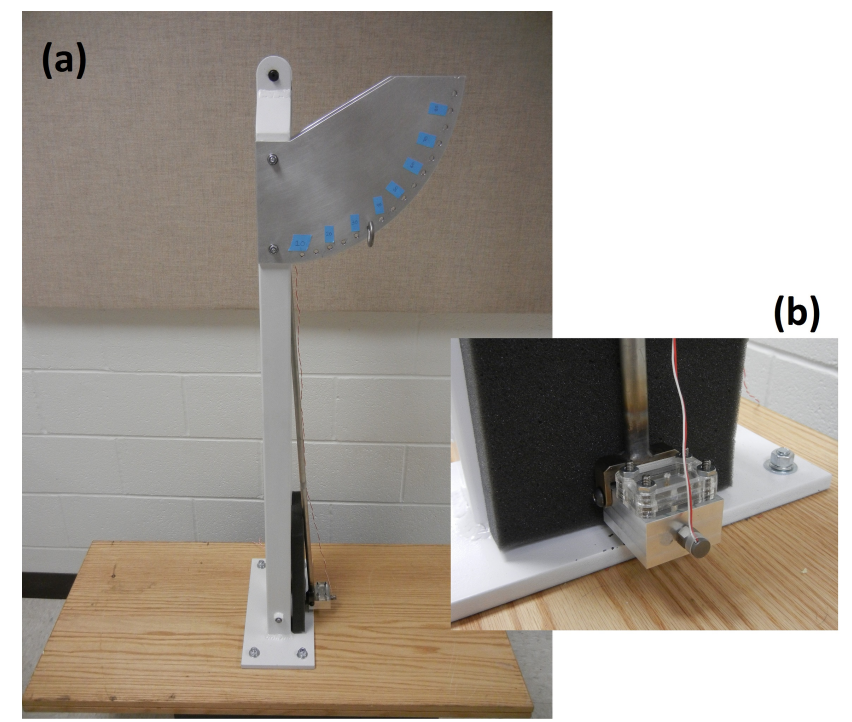

Figure 8. Pendulum and stage used to test switching accelerations under impact conditions. (a) Markers at the top of the pendulum provide an indication of drop angle. (b) This image shows the test stage where the bistable accelerometers are mounted, along with the single-axis accelerometer to measure impact accelerations.

Table 1. Threshold acceleration test results for $8^{\circ}$ configuration, reported in g's.

\begin{tabular}{|c|c|c|c|c|c|c|}
\hline \multirow[b]{2}{*}{ Sample } & \multicolumn{3}{|c|}{ Centrifuge test } & \multicolumn{3}{|c|}{ Impact test } \\
\hline & 1 & 2 & 3 & 1 & 4 & 5 \\
\hline Test 1 & 7.79 & 9.40 & 7.27 & 9.90 & 16.27 & 15.75 \\
\hline 2 & 7.79 & 9.21 & 7.16 & 10.17 & 16.21 & 16.89 \\
\hline 3 & 7.50 & 9.21 & 7.10 & 8.92 & 16.17 & 16.59 \\
\hline 4 & 7.67 & 9.27 & 7.16 & 9.65 & 15.33 & 14.85 \\
\hline \multirow[t]{2}{*}{5} & 7.67 & 9.27 & 7.16 & 10.22 & 16.42 & 15.89 \\
\hline & \multicolumn{6}{|c|}{ After $24 \mathrm{~h}$ in state 2} \\
\hline Test 1 & 7.67 & 9.27 & 7.33 & 9.36 & 15.65 & 15.71 \\
\hline 2 & 7.79 & 9.27 & 7.16 & 8.77 & 15.08 & 16.30 \\
\hline 3 & 7.79 & 9.27 & 7.16 & 8.80 & 17.03 & 15.16 \\
\hline 4 & 7.79 & 9.27 & 7.27 & 8.87 & 16.36 & 16.48 \\
\hline 5 & 7.91 & 9.27 & 7.27 & 8.25 & 16.36 & 15.94 \\
\hline
\end{tabular}

properties, the duration of the impulse will vary. For very rigid materials, the impulse will be sharp and nearly infinitely short in time. For a softer material, the impulse may be smaller in amplitude but have a longer duration. These differences will affect the performance of the threshold accelerometers because in order for switching to occur, not only must the threshold acceleration be reached, but it must be sustained for a sufficient amount of time for the proof mass to shift to the second stable state.

In these tests, a piece of one-inch foam was placed between the rigid stop on the test setup and the swinging stage on the pendulum. This provided impulses that were $35-45 \mathrm{~ms}$

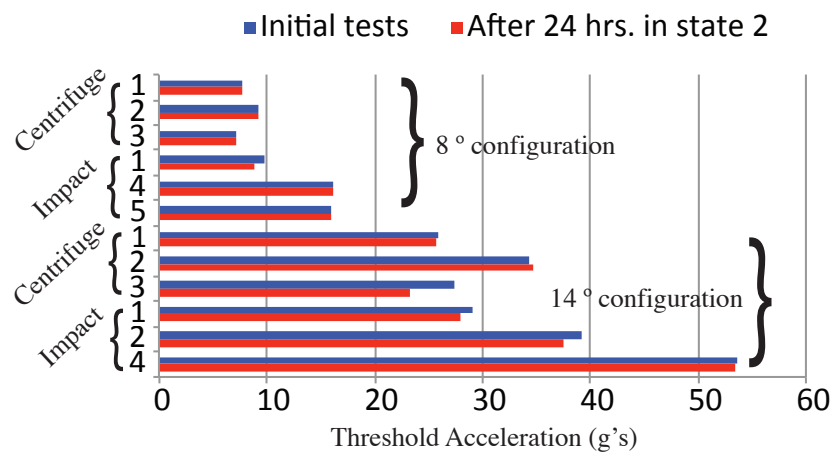

Figure 9. Mean threshold accelerations for each sample, shown for the first set of tests and for the second set, after remaining for $24 \mathrm{~h}$ in the second stable state.

Table 2. Sample means and standard deviations for all $8^{\circ}$ configuration tests, reported in g's.

\begin{tabular}{|c|c|c|c|}
\hline \multicolumn{4}{|c|}{ Centrifuge tests } \\
\hline Sample & 1 & 2 & 3 \\
\hline Mean & 7.69 & 9.27 & 7.17 \\
\hline \pm Std. Dev. & 0.107 & 0.071 & 0.056 \\
\hline \multicolumn{4}{|c|}{ After $24 \mathrm{~h}$ in state 2} \\
\hline Mean & 7.79 & 9.27 & 7.23 \\
\hline \pm Std. Dev. & 0.075 & 0 & 0.068 \\
\hline \multicolumn{4}{|c|}{ Impact tests } \\
\hline Sample & 1 & 4 & 5 \\
\hline Mean & 9.77 & 16.08 & 15.99 \\
\hline \pm Std. Dev. & 0.473 & 0.385 & 0.712 \\
\hline \multicolumn{4}{|c|}{ After $24 \mathrm{~h}$ in state 2} \\
\hline Mean & 8.81 & 16.10 & 15.92 \\
\hline \pm Std. Dev. & 0.353 & 0.670 & 0.465 \\
\hline
\end{tabular}

in duration for the $8^{\circ}$ configuration and $15-25 \mathrm{~ms}$ for the $14^{\circ}$ configuration.

\section{Results}

The results presented here are organized according to flexure beam angle. Tables $1-3$ correspond to results for the $8^{\circ}$ mechanism and Tables $4-6$ correspond to results for the $14^{\circ}$ mechanism. In addition, the mean data from Tables 2 and 5 is shown in the bar chart in Fig. 9 for visual comparison. The "Sample" numbers in each table provide a label for a particular accelerometer. In some cases, an accelerometer that was used in the centrifuge testing remained unbroken and was used for impact testing as well. For example, Sample 1 in Tables 1-3 appear in both centrifuge and impact test results, indicating that it is the same accelerometer.

The measurements shown in Tables 1 and 4 represent the switching acceleration recorded under constant acceleration 
Table 3. $p$ values from paired $t$ test of the means for $8^{\circ}$ configurations.

\begin{tabular}{lccc}
\hline & \multicolumn{3}{c}{ Centrifuge tests } \\
\cline { 2 - 4 } Sample & 1 & 2 & 3 \\
$p$ value & 0.2325 & 0.9972 & 0.0327 \\
\hline & \multicolumn{3}{c}{ Impact tests } \\
\cline { 2 - 4 } $\begin{array}{c}\text { Sample } \\
p \text { value }\end{array}$ & 0.0420 & 0.9712 & 0.8868 \\
\hline
\end{tabular}

Table 4. Threshold acceleration test results for $14^{\circ}$ configuration, reported in g's.

\begin{tabular}{lccccccc}
\hline & \multicolumn{3}{c}{ Centrifuge test } & & \multicolumn{3}{c}{ Impact test } \\
\cline { 2 - 4 } \cline { 6 - 8 } Sample & 1 & 2 & 3 & & 1 & 2 & 4 \\
\hline Test 1 & 25.86 & 33.94 & 28.06 & & 30.58 & 39.51 & 52.87 \\
2 & 25.54 & 34.43 & 27.28 & & 29.27 & 38.40 & 56.13 \\
3 & 25.86 & 34.31 & 27.28 & & 27.05 & 39.08 & 53.98 \\
4 & 25.86 & 34.43 & 27.28 & & 29.71 & 39.53 & 52.47 \\
5 & 25.86 & 34.68 & 27.28 & & 28.37 & 38.87 & 53.89 \\
\hline & & & After 24 h in state 2 & & \\
Test 1 & 25.54 & 34.68 & 23.12 & 28.63 & 38.66 & 52.76 \\
2 & 25.54 & 34.68 & 23.12 & & 28.14 & 34.83 & 54.64 \\
3 & 25.86 & 34.68 & 23.12 & & 27.13 & 37.97 & 53.54 \\
4 & 25.76 & 34.93 & 23.29 & 27.16 & 37.31 & 52.37 \\
5 & 25.54 & 34.68 & Broke & 28.45 & 38.60 & 53.65 \\
\hline
\end{tabular}

conditions on the centrifuge. All results are reported in g's $\left(1 \mathrm{~g}=9.81 \mathrm{~m} \mathrm{~s}^{-2}\right)$. Tables 2 and 5 present the mean and standard deviation for each set of measurements, before and after the $24 \mathrm{~h}$ period.

In order to determine whether there was a statistically significant change in switching acceleration due to stress relaxation, we calculated the p-values from a paired $t$ test on the sample means of each set. We based our analysis of these results on a $95 \%$ confidence interval such that any set with a $p$ value less than five percent was interpreted as one where a change did occur between testing periods. These calculations are presented in Tables 3 and 6 .

\section{Discussion}

The results from these tests suggest that on an individual basis, each accelerometer exhibits very high repeatability. In every test under constant acceleration, the standard deviation was significantly less than $1 \mathrm{~g}$. For impulse acceleration testing, some test sets exhibited a standard deviation slightly larger than $1 \mathrm{~g}$. This may be attributed to the fact that testing on the pendulum offers less precision and repeatability than the centrifuge.
Table 5. Sample means and standard deviations for all $14^{\circ}$ configuration tests, reported in g's.

\begin{tabular}{lccc}
\hline \multicolumn{4}{c}{ Centrifuge tests } \\
\hline Sample & 1 & 2 & 3 \\
Mean & 25.80 & 34.36 & 27.44 \\
\pm Std. Dev. & 0.129 & 0.243 & 0.311 \\
& After 24 h in state 2 \\
\hline Mean & 25.65 & 34.73 & 23.16 \\
\pm Std. Dev. & 0.136 & 0.100 & 0.072 \\
\hline \multicolumn{4}{c}{ Impact tests } \\
\hline Sample & 1 & 2 \\
Mean & 29.00 & 39.08 & 53.57 \\
\pm Std. Dev. & 1.206 & 0.423 & 1.271 \\
\multicolumn{4}{c}{ After 24 h in state 2 } \\
\hline Mean & 27.90 & 37.47 & 53.39 \\
\pm Std. Dev. & 0.638 & 1.410 & 0.786 \\
\hline \multicolumn{4}{c}{} \\
\hline
\end{tabular}

Table 6. $p$ values from paired $t$ test of the means for $14^{\circ}$ configurations.

\begin{tabular}{lccc}
\hline & \multicolumn{3}{c}{ Centrifuge tests } \\
\cline { 2 - 4 } Sample & 1 & 2 & 3 \\
$p$ value & 0.1078 & 0.0397 & 0.0003 \\
\hline & \multicolumn{3}{c}{ Impact tests } \\
\cline { 2 - 4 } Sample & 1 & 2 & 4 \\
$p$ value & 0.1078 & 0.0517 & 0.1420 \\
\hline
\end{tabular}

However, the results also show that among accelerometers of the same configuration, there can be very high variability, particularly among the $14^{\circ}$ configuration accelerometers. This variability can likely be attributed to variations in density and flexural beam thickness from the intended design parameters. For example, growth parameters remained constant for the fabrication of all accelerometers. However, fabrication took place over a period of three to four months because the process requires a significant amount of time from start to finish and growth batches are limited in size. Over this period of time, the controls that regulate the flow of gases to the furnace tend to drift. Additionally, gas supply tanks are occasionally replaced and the composition of the gas can vary somewhat. Both of these variations can have an effect on both density and beam thickness. This will affect the switching acceleration, $a_{\mathrm{s}}$, based on the proportional relations

$a_{\mathrm{s}} \propto \rho$

$a_{\mathrm{s}} \propto t^{3}$

that govern the force required for switching. Each of these variations is being investigated to find ways to reduce the level of uncertainty during growth. 
From Tables 3 and 6 we learned that only four of the tests showed a change in average switching acceleration before and after $24 \mathrm{~h}$ in state 2 , while the other eight showed no statistically significant change. (Here, we consider a $p$ value less than 0.05 to be statistically significant.) Additionally, those accelerometers that did show a change, the change in switching acceleration was greater than $1 \mathrm{~g}$ in only one accelerometer $\left(14^{\circ}\right.$ Configuration, Sample 3$)$. This is a positive indication that even though a slight change may occur due to stress relaxation, the change is quite small relative to the threshold switching acceleration.

\section{Conclusions}

We developed two bistable threshold accelerometer designs and fabricated them using carbon-infiltrated carbon nanotube material. Both designs were tested under constant acceleration and impulse acceleration conditions. During testing, we included a $24 \mathrm{~h}$ wait period in order to measure stress relaxation effects that occur when the accelerometers are in the second stable position for extended periods of time. Results indicate that the accelerometers have very high repeatability individually. Also, our analysis shows that most samples experienced no statistically significant change due to stress relaxation. The accelerometers that did exhibit a change after the $24 \mathrm{~h}$ period showed less than $20 \%$ error relative to the mean initial switching acceleration.

In the future, we intend to investigate different methods of fabrication that may lead to more consistent accelerometers and more reliable testing. For example, as was mentioned previously, handling the accelerometers proved to be a challenge. By developing a process that leaves the accelerometer attached to the silicon substrate so that it is not removed, there will be less risk of breaking during testing. This advantage also lends itself to a better interface between electronic circuits or RF transmitters that can send information to a RF reader regarding the state of the accelerometer. This has been demonstrated previously utilizing surface micromachining techniques with materials other than carbon nanotubes such as plastic, nickel, or silicon dioxide as shown by Todd et al. (2008), Loke et al. (1991), and McNamara and Gianchandani (2004).

\section{Appendix A}

\section{Partial data sets from broken accelerometers}

Table A1. Accelerometers that broke during handling/testing.

\begin{tabular}{lcccc}
\hline \multicolumn{4}{c}{ Switching accelerations reported in g's* } \\
\hline Sample & X1 & X2 & X3 & X4 \\
\hline Test 1 & 40.65 & 19.63 & 41.05 & 42.28 \\
2 & 40.51 & 19.81 & 40.78 & 42.14 \\
3 & 40.38 & 20.38 & 26.95 & 41.87 \\
4 & 40.51 & 20.19 & 25.76 & 42.01 \\
5 & 40.24 & 19.81 & 25.43 & 41.73 \\
\hline
\end{tabular}

After $24 \mathrm{~h}$ in state 2

\begin{tabular}{ccccc}
\hline 1 & 41.05 & - & - & - \\
2 & 40.78 & - & - & - \\
3 & - & - & - & - \\
4 & - & - & - & - \\
5 & - & - & - & - \\
\hline
\end{tabular}

* All samples are $14^{\circ}$ designs and occurred during centrifuge testing.

Acknowledgements. The authors would like to thank Kevin Cole, Equipment Manager for the Department of Mechanical Engineering at Brigham Young University, for his time and expertise which were invaluable in setting up the controls for the experiments.

Edited by: Y. Li

Reviewed by: two anonymous referees

\section{References}

Churaman, W. A., Currano, L., Gee, D., and Zakar, E.: Three-axis MEMS threshold accelerometer switch for enhanced power conservation of MEMS sensors, vol. 54, Acireale, Sicily, Italy, 384 389, 2008.

Edmans, D. M., Gutierrez, A. O., Cormeau, C., Maby, E. W., and Kaufman, H.: Micromachined accelerometer with a movablegate-transistor sensing element, Proc. SPIE, 314-324, 1997.

Fazio, W. C., Lund, J. M., Wood, T. S., Jensen, B. D., Davis, R. C., and Vanfleet, R. R.: Material properties of carbon-infiltrated carbon nanotube-templated structures for microfabrication of compliant mechanisms, in: ASME IMECE 2011, vol. 11, Denver, CO, USA, 481-490, 2011.

Genberg, S. and Pecht, M.: Acceleration sensors for solid state electronic safety and arming devices, Int. J. Hybrid Microelect., 12 126-138, 1989.

Hansen, B., Carron, C., Jensen, B., Hawkins, A., and Schultz, S.: Plastic latching accelerometer based on bistable compliant mechanisms, Smart Mater. Struct., 16, 1967-1972, 2007.

Hutchison, D., Morrill, N., Aten, Q., Turner, B. W., Jensen, B. D., Howell, L., Vanfleet, R., and Davis, R.: Carbon Nanotubes as a Framework for High-Aspect-Ratio MEMS Fabrication, J. Microelectromech. Syst., 19, 75-82, 2010. 
Lassooij, J., Tolou, N., Tortora, G., Caccavaro, S., Menciassi, A., and Herder, J.: A statically balanced and bi-stable compliant end effector combined with a laparoscopic $2 \mathrm{DoF}$ robotic arm, J. Mech. Sci., 3, 85-93, 2012.

Loke, Y., McKinnon, G., and Brett, M.: Fabrication and characterization of silicon micromachined threshold accelerometers, Sensors Actuators A, 29, 235-240, 1991.

Ma, W., Zohar, Y., and Wong, M.: Design and characterization of micro-inertia switches fabricated using low-temperature metalelectroplating technology, vol. 4981, San Jose, CA, USA, 214221, 2003.

McNamara, S. and Gianchandani, Y. B.: LIGA fabricated 19element threshold accelerometer array, Sensors Actuators A, 112, 175-183, 2004.

Rowson, S., Brolinson, G., Goforth, M., Dietter, D., and Duma, S.: Linear and Angular Head Acceleration Measurements in Collegiate Football, J. Biomech. Eng., 131, 061016, doi:10.1115/1.3130454, 2009.

Selvakumar, A., Yazdi, N., and Najafi, K.: A wide-range micromachined threshold accelerometer array and interface circuit, J. Micromech. Microeng., 11, 118-125, 2001.
Sun, X.-Q., Zhou, S., and Carr, W.: A surface micromachined latching accelerometer, in: Solid State Sensors and Actuators, vol. 2, 1997 International Conference on TRANSDUCERS '97, Chicago, 1189-1192, 1997.

Todd, B., Phillips, M., Schultz, S., Hawkins, A., and Jensen, B.: RFID threshold accelerometer, IEEE Autotestcon., 55-59, 2008.

Todd, B., Jensen, B. D., Schultz, S. M., and Hawkins, A. R.: Design and Testing of a Thin-Flexure Bistable Mechanism Suitable for Stamping From Metal Sheets, J. Mech. Design, 132, 071011 , doi:10.1115/1.4001876, 2010.

Yuan, Y., Du, H., and Wang, S.: A miniature in-plane piezoresistive MEMS accelerometer for detection of slider off-track motion in hard disk drives, Microsyst. Technol., 16, 931-940, 2010.

Zhao, J., Jia, J., Wang, H., and Li, W.: A novel threshold accelerometer with postbuckling structures for airbag restraint systems, IEEE Sensors J., 7, 1102-1109, 2007.

Zollo, A., Amoroso, O., Lancieri, M., Wu, Y.-M., and Kanamori, H.: A threshold-based earthquake early warning using dense accelerometer networks, Geophys. J. Int., 183, 963-974, 2010. 